\title{
Reflections from an OECD workshop on environmental benefits of nanotechnology
}

\author{
General issues to consider when doing LCA \\ on nanotechnology-facilitated applications
}

\author{
Frans Møller Christensen
}

Received: 17 September 2009 / Accepted: 20 December 2009/Published online: 22 January 2010

(C) Springer-Verlag 2010

In July 2009, the Organisation for Economic Cooperation and Development (OECD) Working Parties on Nanotechnology and on Manufactured Nano-Materials organised the OECD conference on "Potential environmental benefits of nanotechnology: fostering safe innovation-led growth". The objective of the conference was to provide an opportunity for government, academia and industry to look into stateof-the-art of nanotechnologies, their potential environmental benefits as well as potential health and environmental concerns and to examine related policy considerations.

The conference program covered:

- Two plenary sessions ("Setting the scene" and "Life cycle perspectives")

- Three time slots with eight parallel sessions covering: Water treatment and purification, environmental sensing, clean car technology, cellulose nano fibres, site remediation, better batteries enabled by nano-scale innovation, agricultural nanotechnology, and greener products

- A session with reports from the parallel sessions and final conclusions

The conference gave a good insight into a multitude of nanotechnology applications that could potentially reduce environmental impacts, ranging from applications still in the initial research phase to applications already on or close to the market. Not surprisingly, many discussions circled

F. M. Christensen $(\bowtie)$

Institute for Health and Consumer Protection (IHCP),

Nanobiosciences unit, European Commission,

DG Joint Research Centre (JRC),

TP 202, Via E. Fermi 2749,

21027 Ispra (VA), Italy

e-mail: frans.christensen@ec.europa.eu

URL: http://www.ihcp.jrc.ec.europa.eu around how to weigh these potential benefits against the uncertainties still surrounding the possible toxicity of various types of nano-materials.

Life cycle assessment (LCA) was in general seen as a good tool for comparing these new technologies to already existing technologies and was discussed throughout the individual sessions. See abstracts of the presentations for further details (OECD 2009).

Nano-materials are applied in a significant range of applications and/or to facilitate a long range of technologies (e.g. in electronics as semiconductors; in composite materials to increase strength and lower weight, e.g. of cars; as (carriers to) pharmaceuticals; as coatings for improving surface properties; as catalysts for air and water treatment; in batteries, etc.). Therefore, it does not seem possible to establish an exhaustive list of key issues to consider when doing an LCA involving nanotechnology. However, some general findings on issues to take into account when doing nano-LCAs surfaced throughout the conference, including:

- When looking at alternatives, up-to-date non-nano applications should also be identified and included in the comparative assessment.

- Make sure to include an assessment of scarce materials (like indium, cerium, lithium). This includes inventorying net consumption and an impact assessment taking their scarcity into account. This also involves a careful system definition and/or scenario building in terms of considering to which extent these materials are or can be recycled.

- Remember to include the energy consumption for manufacturing the nano-materials. This has been shown to be sometimes considerable. In addition, energy consumption associated with disposing/recycling the nano applications needs to be carefully addressed. 
- Make sure to inventorise and assess other toxic materials used to produce the nano-materials and other auxiliary chemicals used throughout the life cycle.

- Inventorise and assess emissions of nano-materials (NMs) throughout the life cycle. For example, many NMs go into fixed matrices, but it is important to consider possible loss of nano-particles throughout the life span of the NMs (e.g. due to tear and wear and during final disposal).

- Be honest about what is known about the toxicity and ecotoxicity of the NMs considered. This should be thoroughly discussed, at least qualitatively, in the impact assessment and interpretation part of the LCA.

As noted, these are only some general recommendations, and all nano-LCAs should be framed more carefully. It may, however, be possible to further standardise within certain product families. For example, by making a detailed LCA on one lithium battery application, it may be possible to identify key issues for that application making it possible to streamline subsequent LCAs of lithium battery applications.

For comparison, you may view the proceedings of the workshop "Nanotechnology and Life Cycle Assessment. A Systems Approach to Nanotechnology and Environment" [the workshop was held on 2 to 3 October 2006 (Klöpffer et al. 2007)].

\section{References}

Klöpffer W (Writing team coordinator), Curran MA, Frankl P, Heijungs R, Köhler A, Olson SI (2007) Nanotechnology and Life Cycle Assessment. A systems approach to Nanotechnology and Environment. In: proceedings of the Workshop on Nanotechnology and Life Cycle Assessment, Washington, DC, 2 to 3 October 2006, pp 38, published 20 March 2007. Download: http://cordis.europa.eu/nanotechnology

OECD (2009) Conference programme, background papers and abstracts can be accessed via the conference web site: http:// www.oecd.org/nanobenefits 